\title{
Soluble Urokinase Plasminogen Activator Receptor as a Diagnostic and Prognostic Biomarker in Cardiac Disease
}

\author{
Dimitrios Velissaris ${ }^{\mathrm{a}}$, Nicholas Zareifopoulos ${ }^{\mathrm{b}, \mathrm{f}}$, Ioanna Koniaric ${ }^{\mathrm{c}}$, Vasilios Karamouzos ${ }^{\mathrm{d}}$, \\ Dimitris Bousis ${ }^{\mathrm{b}}$, Andreas Gerakaris ${ }^{\mathrm{b}}$, Christina Platanaki ${ }^{\mathrm{b}}$, Nicholas Kounis ${ }^{\mathrm{e}}$
}

\begin{abstract}
This review summarizes the published literature referring to the use and validity of the biomarker soluble urokinase plasminogen activator receptor (suPAR) when used for the assessment of patients with cardiac diseases. It is measured by enzyme-linked immunosorbent assay (ELISA) in plasma samples. In cardiology a cut-off value range of $3.5-4.5 \mathrm{ng} / \mathrm{mL}$ has been commonly utilized. Different cut-off values may be applied based on the measuring kit used, the patient population and the clinical setting. A PubMed/Medline search was conducted aiming to identify all publications relevant to the use of suPAR in patients with cardiac diseases. A total of 39 studies were included in this review. suPAR as a marker of inflammation has been used more extensively in recent years, alone or in combination with other biomarkers of inflammation and cardiac pathology in the assessment of patients with acute and chronic cardiac diseases. suPAR is closely related to the pathophysiology of cardiac disease, and a number of publications encourages its use as a valuable biomarker in the assessment of patients presenting to the cardiology service. It may be most valuable in the risk assessment of patients with acute coronary syndromes and congestive heart failure, as suPAR elevation may be an independent predictor of mortality in these conditions. In conclusion, among several biomarkers used for clinical entities with underlying inflammatory pathophysiology including cardiac diseases, suPAR is a novel attractive index for the prognostic risk stratification of cardiac patients. More research is warranted to confirm its diagnostic and prognostic validity, alone or combined with other cardiac and inflammatory biomarkers.
\end{abstract}

Manuscript submitted February 23, 2021, accepted March 2, 2021

Published online March 19, 2021

aDepartment of Internal and Emergency Medicine, University of Patras, Patras, Greece

${ }^{b}$ Department of Internal Medicine, University Hospital of Patras, Patras, Greece 'Department of Cardiology, University Hospital of South Manchester NHS Foundation Trust, Manchester, UK

dIntensive Care Unit, University Hospital of Patras, Patras, Greece eDepartment of Cardiology, University of Patras, Patras, Greece

fCorresponding Author: Nicholas Zareifopoulos, Department of Internal Medicine, General University Hospital of Patras, Fifth Floor, Rio Campus, Rion, Patras, Achaea 26504, Greece. Email: nizareifopoulos@gmail.com

doi: https://doi.org/10.14740/jocmr4459
Keywords: Acute coronary syndromes; Biomarker; Cardiac disease; Coronary artery disease; Congestive heart failure; Inflammation; Ischemic heart disease; Myocardial infarction

\section{Introduction}

A complex interplay between genetic and environmental factors is involved in the pathogenesis of most cardiac conditions. Coronary heart disease (CHD) is widely recognized as the most common cause of death worldwide, with substantial morbidity and disability afflicting survivors. The process of the disease is complex and multifactorial, and an underlying chronic inflammation is well recognized. The requirement for timely diagnosis and for more accurate prognostic stratification of patients in cardiology has fueled research into novel cardiac biomarkers, which may be advantageous for this purpose. A chronic elevation of soluble urokinase plasminogen activator receptor (suPAR) may be indicative of chronic endothelial dysfunction and inflammation, an event central to the pathophysiology of CHD and peripheral arterial disease among other manifestations of cardiovascular pathology $[1,2]$. Commonly used serum biomarkers such as the atrial natriuretic peptide (ANP), the brain natriuretic peptide (BNP) which is produced by ventricular cardiomyocytes, and C-reactive protein have been found to be related with adverse cardiovascular events. However, novel biomarkers representing different biological pathways have been evaluated in cardiovascular diseases. Serum levels of the suPAR are close related to the immune and inflammatory activation, and have been used for screening, risk stratification, and monitoring of therapeutic responses in patients with cardiac disease [3].

suPAR is an ubiquitous and non-specific biomarker of inflammation, and a molecule released in response to inflammatory stimuli regardless of the underlying cause. It is a soluble form of the urokinase receptor, a cell membrane receptor with a glycosylphosphatidylinositol (GPI) anchor specific for the urokinase-type plasminogen activator. Urokinase cleaves plasminogen to plasmin, thus activating the fibrinolysis cascade for the dissolution of blood vessel thrombi. suPAR is a $60-\mathrm{kDa}$ glycoprotein released upon cleavage of the GPI anchor; and it is thought to have a chemotactic function, aiding leukocyte 
tissue migration. It is also involved in tissue remodeling after trauma. The measured molecule is the active form of a membrane bound receptor expressed on a variety of cells, including white blood cells, fibroblasts, tissue macrophages, endothelial cells, cardiomyocytes and renal tubular cells [1]. It is excreted in small amounts in urine and saliva, and is measurable in such specimens. Its long plasma half life of 7 - 10 days suggests that it is not readily degraded $[1,2]$. Noxious stimuli induce suPAR release by proteolysis, thus it was assumed that its concentration reflects underlying immune system activity $[4,5]$. It is measured by enzyme-linked immunosorbent assay (ELISA) in blood plasma samples. Two different kits are most commonly used, suPARnostic ${ }^{\circledR}$ ELISA (Virogates) and Quantikine Human UPAR Immunoassay (R\&D Systems), both of which are highly accurate with a threshold of $100 \mathrm{pg} / \mathrm{mL}$ for detection. Values $<3 \mathrm{ng} / \mathrm{mL}$ are generally considered normal, whereas values $>6 \mathrm{mg} / \mathrm{mL}$ are almost always abnormal. Intermediate values are equivocal and the interpretation may differ based on the testing kit used, the study design and the patient population being evaluated. In cardiology a cut-off value range of 3.5 $4.5 \mathrm{ng} / \mathrm{mL}$ has been commonly utilized [1]. In clinical studies various cut-off points have been used to denote different prognostic implications. For example, in the assessment of patients with suspected sepsis in the emergency department a cut-off value of $12 \mathrm{ng} / \mathrm{mL}$ has been used to identify patients with active sepsis at high risk for acute mortality, whereas a value greater than $6 \mathrm{ng} / \mathrm{mL}$ may be used to screen patients at high risk of mortality from non-infectious disease, once infection has been ruled out [5]. Plasma suPAR elevation is associated with systemic inflammatory response syndrome (SIRS), and it is also common in a wide variety of diseases, including cancer, diabetes type II, renal failure, cardiovascular disease and various infections [6-8]. Plasma suPAR concentration has a strong correlation with other markers of inflammation, for example C-reactive protein, tumor necrosis factor- $\alpha$ (TNF- $\alpha)$ and white blood cell count. It is not subject to diurnal variation, with levels remaining constant throughout the day [1]. Women have higher baseline levels of suPAR compared to men, though the reason for this discrepancy remains unclear [9]. The most promising applications of suPAR in cardiology are in the prognostic stratification of patients, as a means to identify which are most likely to deteriorate in a timely manner so as to ensure more adequate monitoring. Due to its exquisitely non-selective nature its value as a diagnostic biomarker for heart conditions is likely to be limited, especially given the wide availability of other far more specific blood tests and imaging studies in cardiology. This is an update of the current literature regarding the role of suPAR as a diagnostic and prognostic biomarker in the field of cardiology.

\section{Methods}

A PubMed/Medline search was conducted in December 2020, and the search terms were: (ischemic heart disease or coronary artery disease or congestive heart failure or myocardial infarction or atrial fibrillation) AND (suPAR or soluble urokinase plasminogen activator receptor). After selection of the most suitable articles to the research object, the bibliographies were reviewed and additional relevant publications were extracted. Articles not available in the English language were excluded. All original studies which could be of clinical relevance on the subject were included in the review. The literature search returned 70 articles which were screened for relevance, reflecting the novel nature of the subject. A total of 39 articles were included in the final review. Two authors (AG and CP) screened the articles for potential relevance, and any disagreements were resolved by consensus. Although this is a narrative review, a systematic review methodology was adhered to during the literature search. The review flow chart is provided (Fig. 1).

\section{Results}

\section{The role of suPAR in acute coronary syndromes}

suPAR has been predominantly utilized as a biomarker in cardiology for the assessment of patients presenting due to acute coronary syndromes, whether as a diagnostic marker to be used alongside cardiac troponin, creatine phosphokinase (CPK) and the bedside electrocardiogram (EKG), or as an indicator of disease severity, prognosis and mortality. In one of the seminal studies on suPAR in cardiology [10], serial CRP and suPAR measurements were obtained on 6-h intervals until peak troponin levels were reached in 296 consecutive ST-elevation myocardial infarction (STEMI) patients admitted for percutaneous coronary intervention (PCI). Mortality and myocardial infarction (MI) recurrence were the primary outcome measures. All-cause mortality was in a range starting from under $10 \%$ for the lowest suPAR quartile up to $41.5 \%$ for the highest. The authors concluded that suPAR may be a reliable predictive factor of mortality and MI recurrence in STEMI patients undergoing PCI. A single-center study which enrolled 449 consecutive chest pain patients investigated whether suPAR retained any prognostic significance in patients presenting with non-STEMI or unstable angina [11]. Long-term follow-up after hospital discharge suggested that the addition of suPAR to troponin and EKG findings improves the prognostic accuracy of both for allcause mortality. The utility of suPAR for the prediction of MI cardiac complications was assessed in another cohort of 139 patients admitted for PCI after their first definite MI episode [12]. Serum levels of suPAR, CRP and maximum troponin T were measured. The cohort was followed up over a single year for MI absolute mortality, subsequent revascularization procedures, MI recurrence and stroke. Reduced glomerular filtration rate and elevated suPAR levels during initial admission functioned as prognostic factors of subsequent morbidity and mortality. However, suPAR levels did not significantly differ between patients with different types of MI, nor between those who subsequently developed heart failure and those who did not. A recent study evaluated the diagnostic value of the combination of suPAR and cardiac troponin in a cohort of patients admitted on suspicion of MI. A total of 1,220 patients were included in the study, of which 245 were definitively diagnosed with MI. Those diagnosed with MI had higher suPAR levels 


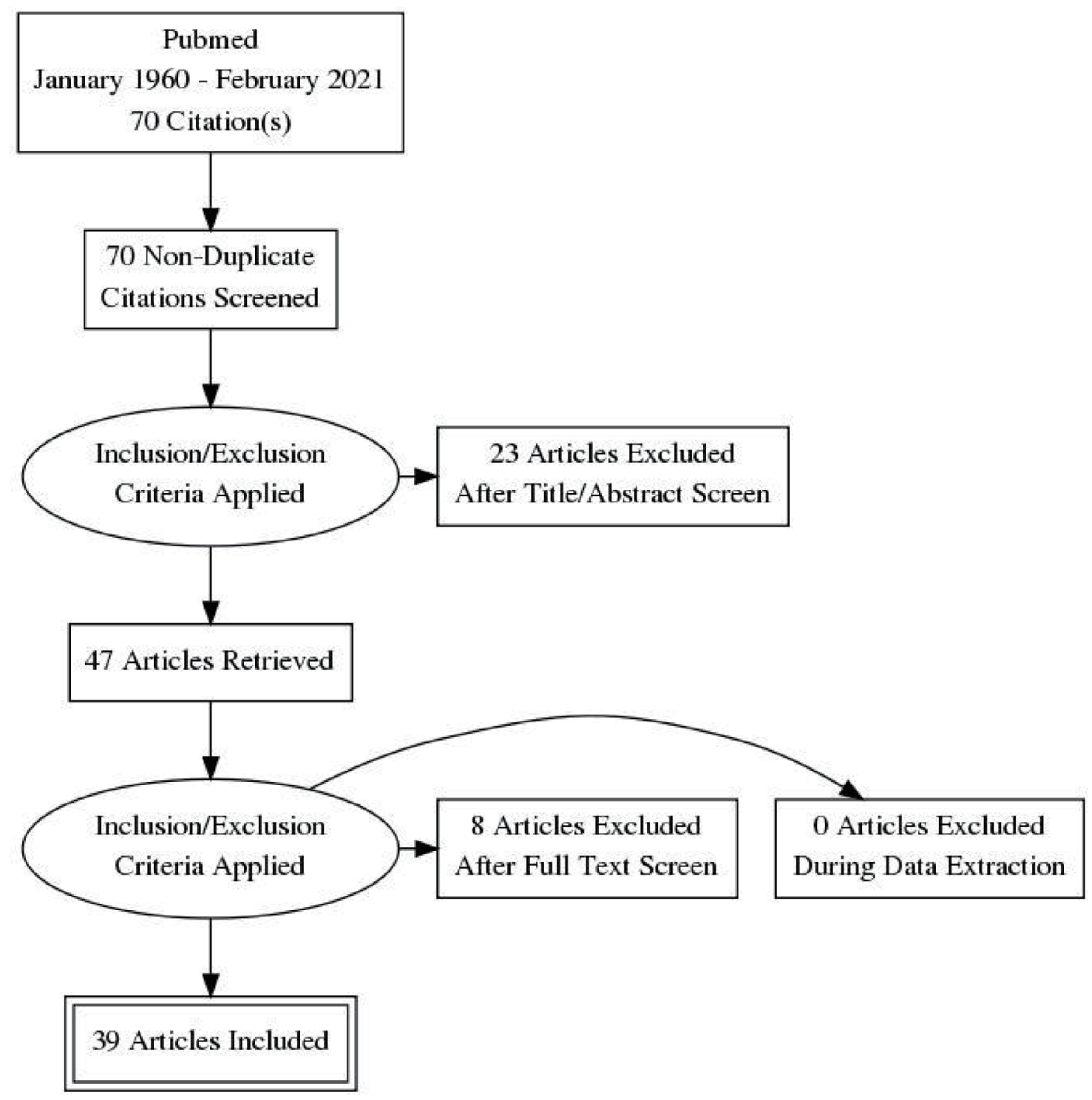

Figure 1. Review flow chart.

on admission compared to those who were not (difference of $0.5 \mathrm{ng} / \mathrm{mL}, \mathrm{P}<0.01)$. However, the combination of troponin and suPAR had reduced sensitivity and specificity compared to troponin alone, casting doubt on the usefulness of suPAR in this setting [13]. The same group evaluated whether suPAR concentration on admission would be predictive of long-term outcomes in a similar cohort. Totally, 1,314 individuals admitted on acute coronary syndrome suspicion were included, of which 308 actually experienced a MI. Admission levels of suPAR correlated well with mortality over 1 year of followup, with performance at 6 months comparable to the Global Registry of Acute Coronary Events (GRACE) score [14]. A retrospective study evaluated suPAR alongside other promising biomarkers including growth-differentiation factor-15 (GDF-15), fetuin A, heart-type fatty acid binding protein $(\mathrm{H}-$ FABP) and soluble suppression of tumorigenicity 2 (sST2) in patients with MI compared to a healthy control group [15].
All biomarkers were elevated in patients with MI compared to controls, with no difference between those with STEMI and those with non-STEMI. The novel biomarkers performed similarly to established biomarkers and clinical measurements, while also being correlated with similar trends in indicators of inflammation such as white blood cell count and CRP. In another study suPAR measurements were obtained in a cohort of 3,367 patients enrolled by the Emory Cardiovascular Biobank and followed up over several years for MI and death of cardiovascular causes [16]. Elevated suPAR levels with a cutoff of $3.5 \mathrm{ng} / \mathrm{mL}$ were associated with an increased risk of MI and cardiovascular mortality. The authors suggested that the addition of suPAR to established models accounting for a variety of cardiac risk factors could slightly increase the prognostic accuracy of these models.

In a particularly vulnerable group, suPAR levels were evaluated in a case-control study of human immunodeficiency 
virus $(\mathrm{HIV})$ patients presenting with first MI $(\mathrm{n}=55)$ and an HIV-positive control group of individuals with no history of cardiac disease $(n=182)$ [17]. The aim of the study was to assess suPAR in the prediction of MI in HIV-1 infected patients. Elevated suPAR was associated with MI even after controlling for possible confounders such as dyslipidemia and viral ribonucleic acid (RNA) titers.

\section{suPAR as a diagnostic and prognostic marker in stable CHD}

Another promising field of research is the use of suPAR for the evaluation of coronary artery disease outside the context of acute coronary syndromes. In the most recent article on this subject, plasma suPAR levels were measured with ELISA technique in two cohorts of 666 asymptomatic individuals and 4,184 stable CHD patients [18]. The study was designed to evaluate sex differences in suPAR production and plasma concentration. The main conclusion was that although baseline suPAR levels are slightly higher in women (in the order of approximately $10 \%$ ), suPAR elevation over the established sex-specific reference range was indicative of an elevated risk for adverse outcomes. Apart from the gender discrepancy in suPAR levels, there may be a racial disparity as well. suPAR levels were measured alongside CRP and $\mathrm{N}$-terminus pro brain natriuretic peptide (NT-proBNP) in a cross-sectional study in an attempt to investigate any correlation between markers of cardiac stress and inflammation in a South African population sample. Baseline levels of all three biomarkers were higher in black compared to white men. NT-proBNP was significantly associated with both CRP and suPAR in black men only. It was concluded that a chronic low-grade inflammatory process as reflected by the combination of an established and a novel biomarker may be indicative of greater cardiovascular risk in black South African men [19]. It is not however clear whether there is a racial difference in baseline suPAR levels, or if suPAR elevation in black compared to white individuals reflects a difference in underlying inflammatory processes. Another group evaluated the role of suPAR in young Chinese patients with a history of angiographically diagnosed CHD [20]. A total of 196 patients below 55 years of age 188 matched healthy controls were enrolled. Active smoking, obesity, age and increased CRP were associated with higher levels of suPAR. Elevation of suPAR above the reference range was associated with CHD after adjustment for confounding variables. In another study [21] plasma concentrations of novel biomarkers reflecting different pathophysiological pathways (sST2 for mechanical heart stress, IGF-BP2 for metabolic derangement, suPAR and GDF-15 for inflammation) were evaluated in patients with stable CHD undergoing exercise regimens. The study's intention was to detect and interpret the effects of training on biomarkers' plasma concentrations. ELISA technique was used in these patients who underwent 4 weeks of high- and moderate-intensity training (EXCITE trial) and in patients with one or more cardiovascular risk factors undergoing 8 months of intensive physical exercise. The 4 weeks of exercise training led to significant changes in the plasma level of sST2 and IGF-BP2, whereas after 8 months of adherence to a high intensity exercise program a significant elevation of IGF-BP2 was detected. Plasma suPAR concentrations did not show any significant change, whereas a significant increase of sST2 after 4 weeks and an increase in IGF-BP2 after 4 weeks and 8 months were reported. In another study suPAR was measured alongside CRP, D-dimers and heat shock protein-70 (hsp70) in a cohort of patients evaluated by coronary angiography $(\mathrm{n}=3,278)[22]$. A composite index was computed based on the number of biomarkers with values above the normal reference range. suPAR elevation with a cut-off value of $3.5 \mathrm{ng} / \mathrm{mL}$ was associated with all-cause death and MI after adjustment for cardiac risk factors and elevated values of the other three biomarkers. Addition of suPAR to a composite index of the other three biomarkers increased prognostic accuracy. The combination of all four tests may be advantageous for the assessment of CHD progression.

suPAR has also been evaluated as a screening test for CHD. The prognostic utility of CRP and suPAR when used alongside the Framingham Risk Score (FRS) was assessed in a Danish cohort of 2,315 individuals [23]. The cohort was followed for over 10 years, and in that time 302 counts of MI, stroke or death from cardiovascular causes were recorded. The most important finding of the study group was that individuals stratified in an intermediate risk category based on the FRS could be downgraded based on lower suPAR and CRP levels, whereas they could be placed in a high risk category if both suPAR and CRP were elevated. Thus suPAR in conjunction with CRP may significantly increase the prognostic accuracy of the FRS in the long-term evaluation of cardiovascular risk.

A prospective cohort study of 1,951 subjects (MONICA study) was designed to evaluate of CRP, NT-proBNP, and suPAR as predictors of cardiovascular morbidity in healthy individuals [24]. Cardiovascular mortality was the primary outcome measure, with the incidence of CHD, congestive heart failure (CHF) and stroke serving as secondary outcome measures. One hundred seventy-seven deaths from cardiovascular causes were recorded over more than 10 years of followup. All three biomarkers were associated with cardiovascular mortality after adjusting for confounders, but only NT-proBNP elevation at baseline was a reliable predictive factor. The investigators concluded that the above biomarkers could serve as prognostic factors of cardiovascular mortality apart from well established risk factors (such as dyslipidemia, diabetes, hypertension and smoking) in the general population. It is however unclear whether suPAR elevation reflects CHD progression or if it is more greatly influenced by activity in other inflammatory foci. Additional attention was drawn to this by a recent study which demonstrated that patients with periodontitis alone and periodontitis comorbid with CHD had elevated plasma and salivary suPAR levels compared with healthy individuals and patients suffering from CHD without periodontitis [25]. A diagnosis of periodontitis predicted plasma suPAR elevation while elevated CRP was the only significant predictor of an increased concentration of suPAR in saliva. It was concluded that in this group suPAR was a useful prognostic indicator in periodontal disease, with further research required to evaluate its role in the assessment of CHD. Another interesting subject is whether suPAR is of use in CHD screening in individuals with comorbidities definitively associated with suPAR eleva- 
tion. One group explored the association between suPAR and the coronary artery calcification score in 99 dialysis-dependent patients with chronic renal failure [26]. A significant association between suPAR levels and CAC score was found. Additionally, 36 individuals experienced at least one cardiovascular event in subsequent years of follow-up. The correlation between suPAR elevation and CAC score may explain why suPAR serves as a prognostic factor of adverse cardiovascular events in this group. The coronary artery calcification index was also utilized in a cross-sectional study of 1,126 individuals in middle age which investigated the relation between CRP, suPAR and the CAC score [27]. The researchers asserted that suPAR was strongly associated with the CAC score, whereas CRP was not, with the association being significant in both sexes but probably greater in females.

A cross-sectional study was designed to investigate a possible role for suPAR as a predictor of coronary microvascular dysfunction. Sixty-six patients with non-obstructive coronary artery disease were included in the study. A significant negative correlation between suPAR and coronary flow reserve was observed, indicating that elevated suPAR levels may be indicative of a defect in coronary microcirculation [28].

\section{suPAR in peripheral artery disease (PAD)}

The role of suPAR in PAD was assessed is of significant interest, as PAD is comorbid with CHD to such a degree that PAD is considered a CHD equivalent. In one such study 51 PAD patients and 55 control subjects without PAD or CHD were included [29]. Measurements for the biomarkers galectin-3, sST2, GDF-15, suPAR, and fetuin-A were obtained by ELISA after blood sampling. Levels of suPAR were significantly elevated in PAD patients, as were all other indices apart from fetuin-A. The important role of suPAR in the prediction of prevalent PAD and PAD-related events was identified in another study [30]. suPAR was measured in 5,810 patients in an attempt to assess its relation to PAD. Plasma suPAR levels were significantly higher in PAD patients compared to individuals with no evidence of PAD, with the degree of suPAR elevation being indicative of PAD severity. Adjusted analysis revealed that elevated suPAR levels were predictive of PAD complications and cardiovascular mortality.

\section{Role of suPAR in the evaluation of heart failure}

Another potential application of suPAR in cardiology is in the evaluation of $\mathrm{CHF}$, where it may correlate with disease progression and function as a predictor of mortality. A recent study examined whether suPAR and other molecules implicated in the fibrinolysis cascade (urokinase-type plasminogen activator, plasminogen activator inhibitor 1 and tissue-type plasminogen activator) could serve to predict adverse outcomes in CHF when measured over time [31]. Two hundred sixty-three patients with CHF were included. All fibrinolysis-associated biomarkers measured were strongly associated with adverse cardiac events commonly encountered in the natural course of the disease. Another group examined the predictive validity of suPAR in outpatients suffering from CHF [32]. The sample consisted of a primary cohort $(n=319)$ and a subsequent validation cohort with the same characteristics $(n=346)$. Elevated levels of suPAR have a strong association with mortality in both groups, suggesting that it may be of value for the prognostic stratification of CHF in the outpatient setting. A study from Japan evaluated a possible for suPAR in the screening for diastolic dysfunction in patients in sinus rhythm with a normal ejection fraction [33]. The study enrolled 291 patients, 26 of which were considered to have diastolic dysfunction. Elevated suPAR was associated diastolic dysfunction even after adjustment for confounders, suggesting a possible role of suPAR screening to prompt further echocardiographic evaluation in this group if such results are validated in a larger sample. The relation between mechanical heart function and suPAR levels was also evaluated in a cohort of patients with diabetes mellitus type $\mathrm{I}(\mathrm{n}=318)$ with no history of cardiac disease and a normal baseline ejection fraction [34]. There was no correlation between suPAR levels and the left ventricular ejection fraction (LVEF) value. Higher suPAR levels were however correlated with measures of hypoclinical diastolic and systolic dysfunction after adjustment for confounders. As type I diabetes is a predisposing factor for development of heart disease, both suPAR and advanced echocardiography seem to be useful diagnostic tools in this population.

Another study investigated the association between suPAR and the incidence of heart failure and atrial fibrillation (AF) in a population-based cohort [35]. suPAR levels were measured in 4,530 members of the Malmo Diet and Cancer study cohort. A total of 109 patients were diagnosed with heart failure, and 321 study participants with AF over more than 15 years of follow-up. It was concluded that suPAR elevation during the study period was independently associated with an increased risk of developing heart failure even after a decade, but it was not a significant predictor for the incidence of AF in the same cohort. One group also investigated the possible role of suPAR in patients with cardiomyopathies [36]. Cardiovascular biomarkers, including H-FABP, sST2, GDF-15 and suPAR were measured in 59 patients with ischemic cardiomyopathy (ICM) and 65 patients with dilative cardiomyopathy (DCM). Plasma samples of all enrolled patients and controls were analyzed with ELISA. Levels of sST2, suPAR and H-FABP were significantly higher in both groups of patients compared to controls. The biomarker values could not however differentiate between the two cardiomyopathies. An inverse correlation between the blood tests and LVEF was noted. A history significant for diabetes mellitus or renal failure as well as simultaneous CRP elevation were associated with higher levels of all evaluated biomarkers. The authors concluded that these novel cardiovascular biomarkers could be useful for more accurate ICM and DCM evaluation, with H-FABP having the greatest potential for widespread clinical use.

\section{SuPAR as a biomarker in AF}

SuPAR may be of value for the evaluation of patients with AF, which may be idiopathic but its prevalence is significant- 
ly higher in individuals with other manifestations of cardiac disease. Two retrospective studies among patients admitted to cardiology department, published in 2017, showed that suPAR was associated with AF, particularly non-paroxysmal AF (NPAF) [37], also was associated with low LVEF and elevated BNP, but not with left ventricular hypertrophy [38].

In another study, suPAR levels were evaluated in a cohort of acutely admitted individuals for the prediction of AF incidence $(n=14,764)$ [39]. AF was diagnosed in $2.4 \%$ of the study samples over 1 year of follow-up. Incident AF was independently associated with elevated suPAR levels on admission, but the utilization of suPAR in conjunction with established models of AF risk did not improve their prognostic accuracy, suggesting minimal clinical value in this application of the emerging biomarker.

\section{suPAR in survivors of cardiac arrest}

An initial pilot study $(\mathrm{n}=55)$ investigated the association between suPAR levels and outcome after cardiac arrest managed by an algorithmic approach which included induced hypothermia [40]. Serial suPAR measurements were obtained up to 72 $\mathrm{h}$ after the induction of hypothermia. An increased concentration of suPAR was associated with prolonged time until spontaneous circulation was restored and increased mortality after 6 months. The findings of another group on this matter were similarly discouraging. A subgroup analysis of the prospective FINNRESUSCI included 287 survivors of out of hospital cardiac arrest [41] in order to examine prognostic value of suPAR in this setting. High suPAR levels after the event were correlated with 90-day mortality, but did not retain their prognostic significance regarding neurological outcomes in survivors after 1 year.

\section{suPAR in the evaluation of aortic valve disease}

Given that suPAR has been utilized as a CHF biomarker, it is not unexpected to evaluate it in aortic valve disease, which comprises the majority of symptomatic valvulopathies and is an important cause of CHF. One study investigated the prognostic value of suPAR in asymptomatic patients with aortic stenosis (AS) [42]. suPAR measurements were available for 1,503 patients enrolled in the Simvastatin and Ezetimibe in Aortic Stenosis (SEAS) study cohort. The investigators reported an association of elevated suPAR with adverse cardiovascular outcomes in non-severe AS. Preoperative plasma suPAR levels were measured in 411 members of the same cohort requiring aortic valve replacement surgery [43]. All patients were asymptomatic with mild to moderate AS. Study endpoints were all-cause mortality, CHF, stroke and renal impairment occurring in the 30 days after operation. Patients with greater preoperative suPAR plasma concentrations were afflicted by a higher incidence of death and complications after the procedure. Investigators in the same study assessed the relation between fibulin-1, a circulating extracellular matrix glycoprotein, the levels of NT-proBNP and suPAR, and explored their interplay in 374 patients with non-severe AS [44]. The authors suggested that fibulin-1 elevation (a putative early diagnostic biomarker of AS and cardiac fibrosis progression) was positively correlated with suPAR concentration.

Another group examined a possible role of suPAR among other emerging cardiac biomarkers in screening patients who underwent a transcatheter aortic valve implantation (TAVI) procedure for postoperative complications [45]. Seventy-nine patients were included. The authors noted an initial surge in suPAR levels in the first few postoperative weeks followed by a return to baseline. Though this is of little prognostic value, the changes in suPAR measurements may be indicative of an inflammatory response associated with the procedure.

\section{Other possible applications of suPAR in cardiology}

Low levels of heart rate variability (HRV) are associated with adverse outcomes after MI and in CHF. One group found an association between increased suPAR and low HRV, with a similar trend being noted for total triglycerides and CRP [46]. A prospective study assessed the possible relation between suPAR, carotid plaque, and the incidence of ischemic stroke and acute coronary syndromes [47]. A total of 5,166 participants in the Malmo Diet and Cancer study (aged 45 - 68 years) were assessed, and ischemic stroke and acute events related to CHD were reported over 15 years of follow-up. suPAR was found to be related with increased occurrence of carotid plaque. Also, suPAR was associated with risk of stroke in multivariate analysis. High levels of suPAR were significantly related to high incidence of risk for ischemic stroke and acute CHD events in patients with carotid plaque formation. One of the first articles published on this matter investigated the association of suPAR with various indicators of subclinical organ dysfunction in a cohort of healthy individuals free of cardiac risk factors $(\mathrm{n}=$ 2,038) [48]. A significant association was found between suPAR and carotid plaque incidence as well as higher urine albu$\mathrm{min} /$ creatinine ratio. After over 10 years of follow-up a weak but significant association between baseline suPAR and the composite endpoint of cardiovascular mortality, MI and stroke emerged, with an adjusted hazard ratio (HR) of 1.16 (1.04 $1.28, \mathrm{P}<0.01)(95 \%$ confidence interval).

All above studies are presented in tabular form in chronological order (Supplementary Material 1, www.jocmr.org).

\section{Discussion}

Given the established role of chronic inflammation in patients with cardiovascular diseases, the identification of serum biomarkers which may aid in early intervention and risk prediction for these diseases has been of increasing importance. suPAR is a molecule associated with immune system and endothelial cell function which may be associated with atherosclerosis and other processes involved in the pathogenesis of cardiovascular disease in which inflammation has been implicated. For this reason several studies have been organized aiming to investigate its role as a diagnostic and prognostic biomarker of 
various cardiovascular diseases and for the timely detection of high-risk cardiovascular patients. Some studies are also in progress assessing whether plasma suPAR measurement can help in the screening, risk stratification and monitoring of therapeutic responses in cardiac diseases. In this review we summarize the major studies referring to the use of suPAR in patients with cardiovascular diseases.

The value of plasma suPAR level was assessed in general population studies. Results from the MONICA study showed that in healthy individuals, among traditional risk factors, biomarkers such as high-sensitivity CRP (hs-CRP), suPAR, and particularly NT-proBNP could serve as predictive and prognostic biomarkers of cardiovascular death [24]. In a study where several biomarkers were assessed, suPAR levels were elevated in patients with PAD [29]. Both suPAR and advanced echocardiography were found useful diagnostic tools for identifying diabetic patients at risk of future clinical cardiac disease [34]. Results from evaluation of 5,166 individuals, participating in the Malmo Diet and Cancer study with a mean follow-up of 15 years for ischemic stroke and CAD showed that suPAR was associated with elevated levels of NT-proBNP and incidence of HF, but not with AF among the middle-aged subjects [35]. Also among healthy individuals a low HRV was related to the immune and inflammatory markers suPAR, CRP and plasma triglyceride [46]; and in the study by Lyngbaek et al when the prognostic value of suPAR and CRP combined with Framingham Risk Score (FRS) were assessed in a healthy population, suPAR had a better prognostication value of cardiovascular disease risk and improved risk prediction when combined with CRP [11]. In the study by Sehestedt et al, which referred to a population cohort of patients without diabetes or prior angiopathy, suPAR was associated with early indicators of organ dysfunction but most importantly functioned as an independent predictor of adverse cardiovascular events [48]. It was even suggested that suPAR to be of useful at the assessment of comorbid CHD and periodontitis [25].

Many of the identified studies are referring to North European and North American populations; however, a crosssectional study assessed inflammatory biomarkers in a mixed white and black bi-ethnic South African population showing that a low-grade inflammation possibly contributed to higher cardiovascular risk in black men, as NT-proBNP, CRP and suPAR levels were higher in the black subgroup [44]. In a study referring to a young Chinese population with CHD, suPAR was identified as an independent risk factor for complications of CHD [20]. Baseline levels of suPAR differ slightly between men and women but the measurements remain valid as long as a different reference range is used for each sex [18].

The prognostic value of suPAR has been investigated in patients with cardiac diseases who underwent invasive procedures such as in those who had a primary PCI after the first MI [12]. Its role as a marker of inflammation for patients after a TAVI procedure [45] has been evaluated, as has its predictive value for postoperative complications in patients who had an aortic valve replacement [43] and its prognostic utility, its temporal course, and its relation to CRP in patients with STsegment elevation MI who had a PCI [10]. In all the aforementioned studies elevated suPAR levels were indicative of increased underlying inflammatory stimuli and were charac- terized by a high predictive value for major cardiac events and complications after invasive procedures.

Its utility was also evaluated in a variety of different settings. Results from a study referring to emergency department patients with suspected acute myocardial infarction (AMI) found that the combination of suPAR and troponin did not facilitate MI diagnosis [13]. It may however be a reliably predictor of mortality in acute coronary syndromes [14]. In patients with chronic renal failure undergoing dialysis, suPAR was associated with increased incidence of adverse cardiovascular events [26]. In stable CHF under evaluation in an outpatient department, elevated suPAR levels were independently predictive of mortality [32].

The biomarker suPAR may also be of value in the evaluation of patients with known chronic cardiac disease. In those with CHF suPAR and other fibrinolysis-associated factors were strongly correlated with adverse cardiac events [31]. suPAR was also useful in the evaluation of patients with ischemic or dilative cardiomyopathy [36]. It retained its prognostic value in patients with AS (SEAS study) [42]; and in a retrospective analysis suPAR plasma levels were elevated in patients with STEMI [15]. In patients who presented in cardiac arrest it was shown in a pilot study that suPAR elevation after hypothermia induction was a predictor of mortality [40], while results from a pre-determined sub-study of the prospective FINNRESUSCI study showed that elevated suPAR levels were associated with poor outcome in out-of-hospital cardiac arrest in ICU patients, and that suPAR alone had inadequate predictive value for poor outcome [41].

It is evident in the literature that suPAR has been examined through several studies for its potential utility in the assessment of patients with cardiac diseases. All relevant studies have been published within the past 10 years. Although suPAR is a non-specific inflammatory biomarker implicated in several diseases of different underlying pathophysiology, it seems to be a promising biomarker with predictive and prognostic value in patients with cardiovascular disease and related critical illness. There are also some fields of research that should be encouraged. First, the research on the correlation of suPAR with other biomarkers related to acute and chronic cardiac diseases, and also in regards to the role of suPAR in different cohorts allowing for comparison between groups of different ethnicity, age and sex is needed. This is required as the samples of the identified studies are not homogenous. Finally, as this molecule has not been extensively used in clinical practice, its validity in different medical settings especially the emergency department should be further evaluated in order to identify whether it would serve as a useful biomarker for the diseases for which it has been evaluated.

\section{Conclusions}

suPAR is a molecule intimately related with the pathophysiology of cardiac diseases; but in the light of the requirement for a biomarker with acceptable diagnostic and prognostic specificity and sensitivity, further research is necessary before the integration of suPAR into daily clinical practice in cardiology. 
In the field of cardiovascular disease, suPAR is more promising as a prognostic indicator for improved accuracy in patient risk stratification than as a purely diagnostic biomarker, echoing a similar sentiment across a wide variety of disciplines. If it were to become routinely available to clinicians, it may be of value for the identification of patients at risk of adverse outcomes when used alongside other laboratory tests, imaging studies and clinical rating scales. It is however necessary to validate the reference range of suPAR in a variety of different settings in this field, and to determine appropriate cut-off values for risk stratification prior to advocating for widespread clinical use.

\section{Supplementary Material}

Suppl 1. Major studies and related findings.

\section{Acknowledgments}

None to declare.

\section{Financial Declaration}

No funding was received for this review.

\section{Conflict of Interest}

The authors declare no conflict of interest.

\section{Author Contributions}

DV did literature search, organized the research, wrote and supervised the paper. $\mathrm{DB}, \mathrm{IK}, \mathrm{AG}, \mathrm{CP}$ did literature search. NZ, $\mathrm{VK}, \mathrm{NK}$ wrote the paper.

\section{Data Availability}

The authors declare that data supporting the findings of this study are available within the article.

\section{References}

1. Thuno M, Macho B, Eugen-Olsen J. suPAR: the molecular crystal ball. Dis Markers. 2009;27(3):157-172.

2. Eugen-Olsen J, Giamarellos-Bourboulis EJ. suPAR: The unspecific marker for disease presence, severity and prognosis. Int J Antimicrob Agents. 2015;46(Suppl 1):S33-34.

3. Hodges GW, Bang CN, Wachtell K, Eugen-Olsen J, Jeppesen JL. suPAR: a new biomarker for cardiovascular disease? Can J Cardiol. 2015;31(10):1293-1302.
4. Velissaris D, Pierrakos C, Karamouzos V, Pantzaris ND, Gogos C. The use of soluble urokinase plasminogen activator receptor (suPAR) as a marker of sepsis in the emergency department setting. A current review. Acta Clin Belg. 2021;76(1):79-84.

5. Velissaris D, Dimopoulos G, Parissis J, Alexiou Z, Antonakos N, Babalis D, Gerakari S, et al. Prognostic role of soluble urokinase plasminogen activator receptor at the emergency department: a position paper by the Hellenic sepsis study group. Infect Dis Ther. 2020;9(3):407-416.

6. Enocsson H, Wettero J, Skogh T, Sjowall C. Soluble urokinase plasminogen activator receptor levels reflect organ damage in systemic lupus erythematosus. Transl Res. 2013;162(5):287-296.

7. Cyrille NB, Villablanca PA, Ramakrishna H. Soluble urokinase plasminogen activation receptor-An emerging new biomarker of cardiovascular disease and critical illness. Ann Card Anaesth. 2016;19(2):214-216.

8. Hayek SS, Sever S, Ko YA, Trachtman H, Awad M, Wadhwani S, Altintas MM, et al. Soluble urokinase receptor and chronic kidney disease. N Engl J Med. 2015;373(20):1916-1925.

9. Backes Y, van der Sluijs KF, Mackie DP, Tacke F, Koch A, Tenhunen JJ, Schultz MJ. Usefulness of suPAR as a biological marker in patients with systemic inflammation or infection: a systematic review. Intensive Care Med. 2012;38(9):1418-1428.

10. Lyngbaek S, Marott JL, Moller DV, Christiansen M, Iversen KK, Clemmensen PM, Eugen-Olsen J, et al. Usefulness of soluble urokinase plasminogen activator receptor to predict repeat myocardial infarction and mortality in patients with ST-segment elevation myocardial infarction undergoing primary percutaneous intervention. Am J Cardiol. 2012;110(12):1756-1763.

11. Lyngbaek S, Andersson C, Marott JL, Moller DV, Christiansen M, Iversen KK, Clemmensen P, et al. Soluble urokinase plasminogen activator receptor for risk prediction in patients admitted with acute chest pain. Clin Chem. 2013;59(11):1621-1629.

12. Wlazel RN, Migala M, Zielinska M, Pawlicki L, RosniakBak K, Szadkowska I. Soluble urokinase plasminogen activator receptor in one-year prediction of major adverse cardiac events in patients after first myocardial infarction treated with primary percutaneous coronary intervention. Arch Med Sci. 2019;15(1):72-77.

13. Sorensen NA, Donmez G, Neumann JT, Nikorowitsch J, Rubsamen N, Blankenberg S, Westermann D, et al. Diagnostic value of soluble urokinase-type plasminogen activator receptor in addition to high-sensitivity troponin I in early diagnosis of acute myocardial infarction. Biomolecules. 2019;9(3);108.

14. Sorensen NA, Nikorowitsch J, Neumann JT, Rubsamen $\mathrm{N}$, Gossling A, Hartikainen TS, Blankenberg S, et al. Predictive value of soluble urokinase-type plasminogen activator receptor for mortality in patients with suspected myocardial infarction. Clin Res Cardiol. 2019;108(12):1386-1393.

15. Schernthaner C, Lichtenauer M, Wernly B, Paar V, Pistulli R, Rohm I, Jung C, et al. Multibiomarker analysis 
in patients with acute myocardial infarction. Eur J Clin Invest. 2017;47(9):638-648.

16. Eapen DJ, Manocha P, Ghasemzadeh N, Patel RS, Al Kassem H, Hammadah M, Veledar E, et al. Soluble urokinase plasminogen activator receptor level is an independent predictor of the presence and severity of coronary artery disease and of future adverse events. J Am Heart Assoc. 2014;3(5):e001118.

17. Rasmussen LJ, Knudsen A, Katzenstein TL, Gerstoft J, Obel N, Jorgensen NR, Kronborg G, et al. Soluble urokinase plasminogen activator receptor (suPAR) is a novel, independent predictive marker of myocardial infarction in HIV-1-infected patients: a nested case-control study. HIV Med. 2016;17(5):350-357.

18. Mehta A, Desai SR, Ko YA, Liu C, Dhindsa DS, Nayak A, Hooda A, et al. Sex differences in circulating soluble urokinase-type plasminogen activator receptor (suPAR) levels and adverse outcomes in coronary artery disease. J Am Heart Assoc. 2020;9(5):e015457.

19. Kruger R, Schutte R, Huisman HW, Hindersson P, Olsen $\mathrm{MH}$, Eugen-Olsen J, Schutte AE. NT-proBNP, C-reactive protein and soluble uPAR in a bi-ethnic male population: the SAfrEIC study. PLoS One. 2013;8(3):e58506.

20. Huang Y, Zhou H, Wu Y, Yang Y, Li W, Lu J, Hu Y. Soluble urokinase plasminogen activator receptor and the risk of coronary artery disease in young Chinese patients. Dis Markers. 2017;2017:4719403.

21. Mirna M, Lichtenauer M, Wernly B, Paar V, Jung C, Kretzschmar D, Uhlemann M, et al. Novel cardiovascular biomarkers in patients with cardiovascular diseases undergoing intensive physical exercise. Panminerva Med. 2020;62(3):135-142.

22. Ghasemzedah N, Hayek SS, Ko YA, Eapen DJ, Patel RS, Manocha P, Al Kassem H, et al. Pathway-specific aggregate biomarker risk score is associated with burden of coronary artery disease and predicts near-term risk of myocardial infarction and death. Circ Cardiovasc Qual Outcomes. 2017;10(3):e001493.

23. Lyngbaek S, Marott JL, Sehestedt T, Hansen TW, Olsen $\mathrm{MH}$, Andersen O, Linneberg A, et al. Cardiovascular risk prediction in the general population with use of suPAR, CRP, and Framingham Risk Score. Int J Cardiol. 2013;167(6):2904-2911.

24. Frary CE, Blicher MK, Olesen TB, Stidsen JV, Greve SV, Vishram-Nielsen JK, Rasmussen SL, et al. Circulating biomarkers for long-term cardiovascular risk stratification in apparently healthy individuals from the MONICA 10 cohort. Eur J Prev Cardiol. 2020;27(6):570-578.

25. Isola G, Polizzi A, Alibrandi A, Williams RC, Leonardi R. Independent impact of periodontitis and cardiovascular disease on elevated soluble urokinase-type plasminogen activator receptor (suPAR) levels. J Periodontol. 2020.

26. Wu W, Cui Y, Hu J, Liao R, Li S, Mo L, Xu L, et al. Soluble urokinase plasminogen activator receptor is associated with coronary artery calcification and cardiovascular disease in patients undergoing hemodialysis. Kidney Blood Press Res. 2018;43(3):664-672.

27. Sorensen MH, Gerke O, Eugen-Olsen J, Munkholm H, Lambrechtsen J, Sand NP, Mickley H, et al. Soluble urokinase plasminogen activator receptor is in contrast to high-sensitive C-reactive-protein associated with coronary artery calcifications in healthy middle-aged subjects. Atherosclerosis. 2014;237(1):60-66.

28. Mekonnen G, Corban MT, Hung OY, Eshtehardi P, Eapen DJ, Al-Kassem H, Rasoul-Arzrumly E, et al. Plasma soluble urokinase-type plasminogen activator receptor level is independently associated with coronary microvascular function in patients with non-obstructive coronary artery disease. Atherosclerosis. 2015;239(1):55-60.

29. Jirak P, Mirna M, Wernly B, Paar V, Thieme M, Betge S, Franz M, et al. Analysis of novel cardiovascular biomarkers in patients with peripheral artery disease. Minerva Med. 2018;109(6):443-450.

30. Samman Tahhan A, Hayek SS, Sandesara P, Hajjari J, Hammadah M, O'Neal WT, Kelli HM, et al. Circulating soluble urokinase plasminogen activator receptor levels and peripheral arterial disease outcomes. Atherosclerosis. 2017;264:108-114.

31. van den Berg VJ, Bouwens E, Umans V, de Maat M, Manintveld OC, Caliskan K, Constantinescu AA, et al. Longitudinally measured fibrinolysis factors are strong predictors of clinical outcome in patients with chronic heart failure: the Bio-SHiFT study. Thromb Haemost. 2019;119(12):1947-1955

32. Koller L, Stojkovic S, Richter B, Sulzgruber P, Potolidis C, Liebhart F, Mortl D, et al. Soluble urokinase-type plasminogen activator receptor improves risk prediction in patients with chronic heart failure. JACC Heart Fail. 2017;5(4):268-277.

33. Fujisaka T, Fujita SI, Maeda D, Shibata K, Takahashi H, Morita H, Takeda Y, et al. Association between suPAR and cardiac diastolic dysfunction among patients with preserved ejection fraction. Heart Vessels. 2017;32(11):1327-1336.

34. Theilade S, Rossing P, Eugen-Olsen J, Jensen JS, Jensen MT. suPAR level is associated with myocardial impairment assessed with advanced echocardiography in patients with type 1 diabetes with normal ejection fraction and without known heart disease or end-stage renal disease. Eur J Endocrinol. 2016;174(6):745-753.

35. Borne Y, Persson M, Melander O, Smith JG, Engstrom $\mathrm{G}$. Increased plasma level of soluble urokinase plasminogen activator receptor is associated with incidence of heart failure but not atrial fibrillation. Eur J Heart Fail. 2014;16(4):377-383.

36. Lichtenauer M, Jirak P, Wernly B, Paar V, Rohm I, Jung $\mathrm{C}$, Schernthaner C, et al. A comparative analysis of novel cardiovascular biomarkers in patients with chronic heart failure. Eur J Intern Med. 2017;44:31-38.

37. Ichihara N, Miyamura M, Maeda D, Fujisaka T, Fujita SI, Morita H, Takeda Y, et al. Association between serum soluble urokinase-type plasminogen activator receptor and atrial fibrillation. J Arrhythm. 2017;33(5):469-474.

38. Fujita SI, Tanaka S, Maeda D, Morita H, Fujisaka T, Takeda Y, Ito T, et al. Serum soluble urokinase-type plasminogen activator receptor is associated with low left ventricular ejection fraction and elevated plasma brain-type natriuretic peptide level. PLoS One. 2017;12(1):e0170546. 
39. Westin O, Rasmussen LJH, Andersen O, Buch E, Olsen JE, Friberg J. Soluble urokinase plasminogen activator receptor (SUPAR) as a predictor of incident atrial fibrillation. J Atr Fibrillation. 2018;10(6):1801.

40. Rundgren M, Lyngbaek S, Fisker H, Friberg H. The inflammatory marker suPAR after cardiac arrest. Ther Hypothermia Temp Manag. 2015;5(2):89-94.

41. Jalkanen V, Vaahersalo J, Pettila V, Kurola J, Varpula T, Tiainen M, Huhtala H, et al. The predictive value of soluble urokinase plasminogen activator receptor (SuPAR) regarding 90-day mortality and 12-month neurological outcome in critically ill patients after out-of-hospital cardiac arrest. Data from the prospective FINNRESUSCI study. Resuscitation. 2014;85(11):1562-1567.

42. Hodges GW, Bang CN, Eugen-Olsen J, Olsen MH, Boman K, Ray S, Gohlke-Barwolf C, et al. SuPAR predicts cardiovascular events and mortality in patients with asymptomatic aortic stenosis. Can J Cardiol. 2016;32(12):14621469.

43. Hodges GW, Bang CN, Eugen-Olsen J, Olsen MH, Boman K, Ray S, Kesaniemi AY, et al. SuPAR predicts postoperative complications and mortality in patients with asymptomatic aortic stenosis. Open Heart. 2018;5(1):e000743.

44. Kruger R, Rasmussen LM, Argraves WS, Eugen-Olsen
J, Nielsen OW, Blyme A, Willenheimer R, et al. Extracellular matrix biomarker, fibulin-1, is closely related to NT-proBNP and soluble urokinase plasminogen activator receptor in patients with aortic valve stenosis (the SEAS study). PLoS One. 2014;9(7):e101522.

45. Mirna M, Wernly B, Paar V, Jung C, Jirak P, Figulla HR, Kretzschmar D, et al. Multi-biomarker analysis in patients after transcatheter aortic valve implantation (TAVI). Biomarkers. 2018;23(8):773-780.

46. Intzilakis T, Hartmann G, Mouridsen MR, Eugen-Olsen J, Kumarathurai P, Madsbad S, Almdal TP, et al. Soluble urokinase plasminogen activator receptor, C-reactive protein and triglyceride are associated with heart rate variability in non-diabetic Danes. Eur J Clin Invest. 2013;43(5):457-468.

47. Persson M, Ostling G, Smith G, Hamrefors V, Melander O, Hedblad B, Engstrom G. Soluble urokinase plasminogen activator receptor: a risk factor for carotid plaque, stroke, and coronary artery disease. Stroke. 2014;45(1):18-23.

48. Sehestedt T, Lyngbaek S, Eugen-Olsen J, Jeppesen J, Andersen O, Hansen TW, Linneberg A, et al. Soluble urokinase plasminogen activator receptor is associated with subclinical organ damage and cardiovascular events. Atherosclerosis. 2011;216(1):237-243. 